\title{
CHARACTERIZATION OF THE MARGINAL DISTRIBUTIONS OF MARKOV PROCESSES USED IN DYNAMIC RELIABILITY
}

\author{
CHRISTIANE COCOZZA-THIVENT, ROBERT EYMARD, SOPHIE MERCIER, \\ AND MICHEL ROUSSIGNOL
}

Received 3 May 2004; Revised 15 February 2005; Accepted 22 February 2005

In dynamic reliability, the evolution of a system is described by a piecewise deterministic Markov process $\left(I_{t}, X_{t}\right)_{t \geq 0}$ with state-space $E \times \mathbb{R}^{d}$, where $E$ is finite. The main result of the present paper is the characterization of the marginal distribution of the Markov process $\left(I_{t}, X_{t}\right)_{t \geq 0}$ at time $t$, as the unique solution of a set of explicit integro-differential equations, which can be seen as a weak form of the Chapman-Kolmogorov equation. Uniqueness is the difficult part of the result.

Copyright (c) 2006 Christiane Cocozza-Thivent et al. This is an open access article distributed under the Creative Commons Attribution License, which permits unrestricted use, distribution, and reproduction in any medium, provided the original work is properly cited.

\section{Introduction}

In dynamic reliability, a system is considered, which evolves in time according to a twocomponent Markov process $\left(I_{t}, X_{t}\right)_{t \geq 0}$ : the symbol $I_{t}$ corresponds to some physical state of the system and has a finite state-space $E$, whereas $X_{t}$ represents some "environmental" condition (temperature, pressure, ...) and takes its values in $\mathbb{R}^{d}$. The transition rate between two different states $i$ and $j$ of $E$ may vary with the current environmental condition $x$ in $\mathbb{R}^{d}$. The transition rate from state $i$ to state $j$ then appears as a function from $\mathbb{R}^{d}$ to $\mathbb{R}_{+}$, and will be denoted by $a(i, j, \cdot)$. The evolution of the environmental component $X_{t}$ is described by a set of differential equations, which depend on the physical state of the item. More precisely, the dynamic of $X_{t}$ is such that, given $I_{t}(\omega)=i$ for all $t \in[a, b]$, we have

$$
\frac{d}{d t} X_{t}(\omega)=\mathbf{v}\left(i, X_{t}(\omega)\right)
$$

for all $t \in[a, b]$, where $\mathbf{v}$ is a mapping from $E \times \mathbb{R}^{d}$ to $\mathbb{R}^{d}$. As a consequence, the trajectory $\left(X_{t}\right)_{t \geq 0}$ is deterministic between any two consecutive jumps of $\left(I_{t}\right)_{t \geq 0}$, and $\left(X_{t}\right)_{t \geq 0}$ is the 
solution of the ordinary differential equation $d y / d t=\mathbf{v}(i, y)$. Such processes are called piecewise deterministic Markov processes, see Davis [7, 8].

In dynamic reliability, the process $\left(X_{t}\right)_{t \geq 0}$ is usually assumed to be continuous so that no jump is allowed for the environmental component (see [12], e.g., and other papers by P. E. Labeau). In the present paper, this assumption of continuity is relaxed and the process $\left(X_{t}\right)_{t \geq 0}$ may jump simultaneously with the process $\left(I_{t}\right)_{t \geq 0}$. More precisely, when the process $I_{t}$ jumps from state $i$ to state $j$, we assume that the process $\left(X_{t}\right)$ belongs to $(y, y+d y)$ with probability $\mu(i, j, x)(d y)$ at the jump time, given that $X_{t}=x$ just before the jump (where $(i, j, x) \in E^{2} \times \mathbb{R}^{d}$ ). In the case $\mu(i, j, x)(d y)=\delta_{x}(d y)$ (for all $i, j, x$ ), the process $\left(X_{t}\right)_{t \geq 0}$ is continuous and our model meets the usual one. Our model however includes a lot of other interesting cases such as interacting semi-Markov processes or non-homogenous Markov processes.

The marginal distribution of the process $\left(I_{t}, X_{t}\right)_{t \geq 0}$ at time $t$ usually is a complicated characteristic which is not analytically reachable (see, e.g., [12]). The aim of the present paper is to characterize such a marginal distribution as the unique measure solution of a set of explicit integro-differential equations. Such equations can actually be seen as a weak form of the Chapman-Kolmogorov (CK) equations associated to the Markov process $\left(I_{t}, X_{t}\right)_{t \geq 0}$. In a second paper (see [3]), we use such a characterization to propose a numerical algorithm, which is shown to converge weakly towards the right marginal distribution in a third paper [4].

Piecewise deterministic Markov processes (PDMP) have already been studied by Davis [8] (other references therein). However, the main part of the present work, namely, existence and uniqueness of solutions to the CK equation is not treated by Davis, who is mainly interested in functionals of the process which do not englobe marginal distributions. PDMP have also been studied by Costa, Dufour, and Raymundo in $[5,6,9]$ and by Borovkov and Novikov in [2], with different objectives from ours. A chapter in [13] is also devoted to PDMP.

The paper is organized as follows: notations and assumptions are given in Section 2.

In Section 3, the process $\left(I_{t}, X_{t}\right)_{t \geq 0}$ is constructed, using the Markov renewal theory. We derive that $\left(I_{t}, X_{t}\right)_{t \geq 0}$ is a Markov process and give an explicit expression for its transition probabilities and for its full generator on regular functions.

In Section 4, we first give some weak form for the CK equations with regular test functions. The existence of a solution to such equations is ensured by the construction of Section 3. Then we show the uniqueness of the solution by extending the set of test functions to more general set of functions. This is the main part of the paper.

Concluding remarks are given in Section 5.

\section{Notations-assumptions}

We use the following notations.

(1) Symbol $E$ stands for the finite state-space of the studied item.

(2) For $d \in \mathbb{N}^{*}$, symbols $\mathscr{P}\left(\mathbb{R}^{d}\right)$ and $\mathscr{P}\left(E \times \mathbb{R}^{d}\right)$, respectively, represent the set of probability measures on $\mathbb{R}^{d}$ and $E \times \mathbb{R}^{d}$.

(3) Symbols $C_{b}(F, \mathbb{R})$ and $C^{1}(F, \mathbb{R})$, respectively, stand for the set of bounded continuous functions and the set of continuously differentiable functions from a 
Banach space $F$ to $\mathbb{R}$ (or $\mathbb{R}_{+}$). In the following, we use $F=\mathbb{R}^{d}, F=\mathbb{R}^{d} \times \mathbb{R}$, $F=\mathbb{R}^{d} \times[0, T], C_{b}^{1}(F, \mathbb{R})=C_{b}(F, \mathbb{R}) \cap C^{1}(F, \mathbb{R})$. Symbol $C_{c}^{1}(F, \mathbb{R})$ stands for the set of continuously differentiable functions with a compact support.

(4) For any set $F$, symbols $F^{E}$ and $F^{E \times E}$, respectively, represent the set of functions

$$
f:\left\{\begin{array}{l}
E \longrightarrow F, \\
i \longmapsto f(i),
\end{array} \quad f:\left\{\begin{array}{l}
E \times E \longrightarrow F, \\
(i, j) \longmapsto f(i, j) .
\end{array}\right.\right.
$$

For instance, $C_{b}\left(\mathbb{R}^{d}, \mathbb{R}\right)^{E}$ stands for the set of functions $f: E \rightarrow C_{b}\left(\mathbb{R}^{d}, \mathbb{R}\right)$, namely, such that $f(i)$ is a bounded continuous function from $\mathbb{R}^{d}$ to $\mathbb{R}$, for all $i \in E$. For the sake of simplicity, we write $f(i, x)$ or $f(i, j, x)$ instead of $(f(i))(x)$ or $(f(i, j))(x)$, for $i, j \in E$ and $x$ in $\mathbb{R}^{d}$ or $\mathbb{R}^{d} \times \mathbb{R}$.

With these notations, we adopt the following assumptions, which will be referred to as assumptions $(H)$.

(1) The transition rate $a$ is assumed to be such that $a \in C_{b}\left(\mathbb{R}^{d}, \mathbb{R}_{+}\right)^{E \times E}$, namely, nonnegative, continuous, and bounded by some real number $A>0$.

(2) The velocity $\mathbf{v}$ which appears in (1.1) is assumed to be such that:

(i) $\mathbf{v} \in C\left(\mathbb{R}^{d}, \mathbb{R}^{d}\right)^{E}$,

(ii) the function $\mathbf{v}(i, \cdot)$ is locally Lipschitz continuous (for all $i \in E$ ),

(iii) the function $\operatorname{div} \mathbf{v}(i, \cdot)$ is almost everywhere bounded by some real value $D>0$ (for all $i \in E$ ),

(iv) the function $\mathbf{v}(i, \cdot)$ is sublinear (for all $i \in E$ ): there are some $V_{1}>0$ and $V_{2}>0$ such that

$$
\forall i \in E, \forall x \in \mathbb{R}^{d}, \quad\|\mathbf{v}(i, x)\| \leq V_{1}\|x\|+V_{2}
$$

(3) The distribution $\{\mu(i, j, x)\}$ which controls the jumps of $\left(X_{t}\right)_{t \geq 0}$ by jump times of $\left(I_{t}\right)_{t \geq 0}$ is such that, for $i, j \in E$ and $\psi \in C_{b}\left(\mathbb{R}^{d}, \mathbb{R}\right)$, the function $x \rightarrow \int \psi(y) \mu(i, j$, $x)(d y), x \in \mathbb{R}^{d}$, is continuous (where $\mu: E \times E \times \mathbb{R}^{d} \rightarrow \mathscr{P}\left(\mathbb{R}^{d}\right)$ ).

These assumptions guarantee the existence and uniqueness of the solution to the differential equations fulfilled by the environmental component. They also provide us with some classical properties for the solution which are summed up in the following lemma.

Lemma 2.1. There exists one and only one function $g \in C\left(\mathbb{R}^{d} \times \mathbb{R}, \mathbb{R}^{d}\right)^{E}$ of the class $C^{1}$ with respect to time $t$ such that for any $i \in E$,

$$
\frac{\partial g}{\partial t}(i, x, t)=\mathbf{v}(i, g(i, x, t)), \quad \forall(x, t) \in \mathbb{R}^{d} \times \mathbb{R}
$$

with an initial condition

$$
g(i, x, 0)=x, \quad \forall x \in \mathbb{R}^{d}
$$


Moreover, this unique solution $g$ fulfils the following properties.

(1) The function $x \mapsto g(i, x, t)$ is locally Lipschitz continuous with respect to $x$ on $\mathbb{R}^{d}$, for all $t>0$ and $i \in E$.

(2) For all $x \in \mathbb{R}^{d},(s, t) \in \mathbb{R} \times \mathbb{R}$, and $i \in E$, one has

$$
g(i, g(i, x, s), t)=g(i, x, s+t)
$$

\section{Probabilistic construction of the process}

In this section, we first construct the process $\left(I_{t}, X_{t}\right)_{t \geq 0}$ in a similar way as in Davis [8]. We then derive explicit expressions for the transition probabilities of the process $\left(I_{t}, X_{t}\right)_{t \geq 0}$ and for its full generator on the class of regular functions.

3.1. Construction of the process. In order to construct the process $\left(I_{t}, X_{t}\right)_{t \geq 0}$ with values in $E \times \mathbb{R}^{d}$, we first define a Markov renewal process $\left(T_{n},\left(I_{T_{n}}, X_{T_{n}}\right)\right)_{n \geq 0}$ by its conditional distributions. Let us denote by $Q\left(I_{T_{n}}, X_{T_{n}}, j, d y, d u\right)$ the conditional distribution of $\left(I_{T_{n+1}}, X_{T_{n+1}}, T_{n+1}-T_{n}\right.$ ), given $\left(T_{k},\left(I_{T_{k}}, X_{T_{k}}\right)\right)_{k \leq n}$ (or equivalently given $\left(I_{T_{n}}, X_{T_{n}}\right)$ ). Setting $\lambda(i, \cdot)=\sum_{j \in E} a(i, j, \cdot)$ for $i \in E$, we define $Q$ in the following way:

$$
\begin{aligned}
& \mathbf{E}\left[h\left(X_{T_{n+1}}, T_{n+1}-T_{n}\right) \mathbf{1}_{\left\{I_{T_{n+1}}\right\}} \mid\left(T_{k},\left(I_{T_{k}}, X_{T_{k}}\right)\right)_{k \leq n}\right] \\
& =\int_{0}^{\infty} \exp \left(-\int_{0}^{u} \lambda\left(I_{T_{n}}, g\left(I_{T_{n}}, X_{T_{n}}, v\right)\right) d v\right) a\left(I_{T_{n}}, j, g\left(I_{T_{n}}, X_{T_{n}}, u\right)\right) \\
& \quad \times\left(\int h(y, u) \mu\left(I_{T_{n}}, j, g\left(I_{T_{n}}, X_{T_{n}}, u\right)\right)(d y)\right) d u \\
& =\int_{0}^{\infty} h(y, u) Q\left(I_{T_{n}}, X_{T_{n}}, j, d y, d u\right) .
\end{aligned}
$$

Here, $h$ is any positive measurable function on $\mathbb{R}^{d} \times \mathbb{R}_{+}$and $j \in E$.

The process $\left(I_{t}, X_{t}\right)_{t \geq 0}$ is now constructed as

$$
\text { if } T_{n} \leq t<T_{n+1} \text {, then } I_{t}=I_{T_{n}}, \quad X_{t}=g\left(I_{T_{n}}, X_{T_{n}}, t-T_{n}\right) \text {. }
$$

As noted in Section 1, we can see that, given $\left(I_{T_{n}}, X_{T_{n}}\right)_{n \geq 0}$, the trajectory of $\left(X_{t}\right)_{t \geq 0}$ is purely deterministic on $\left[T_{n}, T_{n+1}\right)$ for $n=0,1,2, \ldots$, with $T_{0}=0$.

3.2. Transition probabilities. From the previous construction, we derive an explicit expression for the transition probabilities of $\left(I_{t}, X_{t}\right)_{t \geq 0}$. This is based on calculations of conditional expectations, using several properties of the Markov renewal process $\left(T_{n},\left(I_{T_{n}}\right.\right.$, $\left.\left.X_{T_{n}}\right)\right)_{n \geq 0}$ such as

$$
\begin{aligned}
\mathbf{E} & {\left[h\left(T_{n+1}-T_{n}\right) \mid\left(T_{k},\left(I_{T_{k}}, X_{T_{k}}\right)\right)_{k \leq n}\right] } \\
& =\int_{0}^{\infty} h(u) \exp \left(-\int_{0}^{u} \lambda\left(I_{T_{n}}, g\left(I_{T_{n}}, X_{T_{n}}, v\right)\right) d v\right) \lambda\left(I_{T_{n}}, g\left(I_{T_{n}}, X_{T_{n}}, u\right)\right) d u .
\end{aligned}
$$


It is convenient to introduce the following notation:

$$
c_{i}(t, x)=\exp \left(-\int_{0}^{t} \lambda(i, g(i, x, v)) d v\right)
$$

Proposition 3.1. The process $\left(I_{t}, X_{t}\right)_{t \geq 0}$ is a homogeneous Markov process with values in $E \times \mathbb{R}^{d}$. Its transition probabilities $P_{t}(i, x, j, d y)$ are given by

$$
\begin{aligned}
\int h(y) & P_{t}(i, x, j, d y) \\
= & 1_{\{i=j\}} c_{i}(t, x) h(g(i, x, t)) \\
& +\int_{0}^{t} c_{i}(s, x) a(i, j, g(i, x, s))\left(\int c_{j}(t-s, y) h(g(j, y, t-s)) \mu(i, j, g(i, x, s))(d y)\right) d s \\
& +\sum_{p \geq 2} \sum_{j_{1}, \ldots, j_{p-1}} \int_{\left\{s_{1} \geq 0, \ldots, s_{p} \geq 0, s_{1}+\cdots+s_{p} \leq t\right\}} \cdots s_{1} \cdots d s_{p} \\
& \times c_{i}\left(s_{1}, x\right) a\left(i, j_{1}, g(i, x, s)\right) \int \mu\left(i, j_{1}, g\left(i, x, s_{1}\right)\right)\left(d y_{1}\right) \\
& \times \prod_{i=1}^{p-2} c_{j_{i}}\left(s_{i+1}, y_{i}\right) a\left(j_{i}, j_{i+1}, g\left(j_{i}, y_{i}, s_{i+1}\right)\right) \int \mu\left(j_{i}, j_{i+1}, g\left(j_{i}, y_{i}, s_{i+1}\right)\right)\left(d y_{i+1}\right) \\
& \times c_{j_{p-1}}\left(s_{p}, y_{p-1}\right) a\left(j_{p-1}, j, g\left(j_{p-1}, y_{p-1}, s_{p}\right)\right) \int \mu\left(j_{p-1}, j, g\left(j_{p-1}, y_{p-1}, s_{p}\right)\right)(d y) \\
& \times c_{j}\left(t-\left(s_{1}+\cdots+s_{p}\right), y\right) h\left(g\left(j, y, t-\left(s_{1}+\cdots+s_{p}\right)\right)\right),
\end{aligned}
$$

where $h$ is any bounded measurable function.

Remark 3.2. These transition probabilities are the sum of different terms. The first term corresponds to the case of no jump between 0 and $t$, and consequently to a deterministic trajectory on $[0, t]$. The second term corresponds to the case of one single jump, and the other terms to more than two jumps.

As usual, the transition probabilities $P_{t}$ can be seen as operators on the set of measurable bounded functions defined on $E \times \mathbb{R}^{d}$ in the following way:

$$
\forall(i, x) \in E \times \mathbb{R}^{d}, \quad P_{t} h(i, x)=\mathbf{E}\left(h\left(I_{t}, X_{t}\right) \mid I_{0}=i, X_{0}=x\right)=\sum_{j \in E} \int h(j, y) P_{t}(i, x, j, d y) .
$$

The following two corollaries can be easily checked thanks to the expression of $P_{t}$ as given by Proposition 3.1. 
Corollary 3.3. Let $h \in C_{b}\left(\mathbb{R}^{d}, \mathbb{R}\right)^{E}$, then for all $t \in \mathbb{R}_{+}, P_{t} h \in C_{b}\left(\mathbb{R}^{d}, \mathbb{R}\right)^{E}$.

Corollary 3.4. For $h \in C_{b}\left(\mathbb{R}^{d}, \mathbb{R}\right)^{E}$ and $\left(i_{0}, x_{0}\right) \in E \times \mathbb{R}^{d}, t \rightarrow P_{t} h\left(i_{0}, x_{0}\right), t \in \mathbb{R}_{+}$, is a continuous function.

3.3. Generator. We now exhibit sufficient conditions for a function $h$ to be in the domain of the full generator $\mathscr{L}$ of the Markov process $\left(I_{t}, X_{t}\right)_{t \geq 0}$, and we give the expression of $\mathscr{L} h$ for such an $h$. Alternative sufficient conditions may be found in [13, Theorem 11.2.2] or [8]. In all the following, we will use the set $D_{0}$ such that

$$
D_{0}=\left\{h: h \in C_{b}^{1}\left(\mathbb{R}^{d}, \mathbb{R}\right)^{E}, \mathbf{v}(i, \cdot) \cdot \nabla h(i, \cdot) \in C_{b}\left(\mathbb{R}^{d}, \mathbb{R}\right), \forall i \in E\right\}
$$

Here, $\nabla$ represents the gradient with respect to the second variable (in $\mathbb{R}^{d}$ ) and

$$
\mathbf{v}(i, \cdot) \cdot \nabla h(i, \cdot)=\sum_{k=1}^{d} \frac{\partial h}{\partial x_{k}}(i, \cdot) \mathbf{v}^{(k)}(i, \cdot)
$$

with $\mathbf{v}(i, \cdot)=\left(\mathbf{v}^{(1)}(i, \cdot), \mathbf{v}^{(2)}(i, \cdot), \ldots, \mathbf{v}^{(d)}(i, \cdot)\right)$.

Proposition 3.5. For $h \in D_{0}$, let us define $\mathscr{L} h$ in the following way:

$$
\begin{aligned}
\mathscr{L} h(i, x) & =\sum_{j} a(i, j, x) \int h(j, y) \mu(i, j, x)(d y)-h(i, x) \lambda(i, x)+\sum_{k=1}^{d} \frac{\partial h}{\partial x_{k}}(i, x) \mathbf{v}^{(k)}(i, x) \\
& =\sum_{j} a(i, j, x) \int(h(j, y)-h(i, x)) \mu(i, j, x)(d y)+\mathbf{v}(i, x) \cdot \nabla h(i, x) .
\end{aligned}
$$

Then, under assumptions $(H), h \in D_{0}$ belongs to the domain of the full generator $\mathscr{L}$ of the Markov process $\left(I_{t}, X_{t}\right)_{t \geq 0}$. Equivalently, $h\left(I_{t}, X_{t}\right)-h\left(I_{0}, X_{0}\right)-\int_{0}^{t} \mathscr{L} h\left(I_{s}, X_{s}\right) d s$ is a martingale with respect to the natural filtration of the process $\left(I_{t}, X_{t}\right)_{t \geq 0}$.

Proof. Assumptions $(H)$ imply that $\mathscr{L}$ is a linear operator from $D_{0}$ to the set of bounded measurable functions. For $(i, x) \in E \times \mathbb{R}^{d}$ and $h \in D_{0}$, let us consider

$$
h_{t}(i, x)=\frac{P_{t} h(i, x)-h(i, x)}{t}=\frac{\sum_{j \in E} \int h(j, y) P_{t}(i, x, j, d y)-h(i, x)}{t}
$$

Due to classical arguments, it is sufficient to prove that $\lim _{t \rightarrow 0_{+}} h_{t}(i, x)=\mathscr{L} h(i, x)$, and that $h_{t}(i, x)$ is uniformly bounded for $(i, x) \in E \times \mathbb{R}^{d}$ and $t$ in a neighborhood of 0 .

Based on Proposition 3.1, we have

$$
P_{t} h(i, x)-h(i, x)=A_{1}+A_{2}+A_{3}
$$


where

$$
\begin{aligned}
A_{1}= & c_{i}(t, x) h(i, g(i, x, t))-h(i, x), \\
A_{2}= & \sum_{j} \int_{0}^{t} c_{i}(s, x) a(i, j, g(i, x, s)) \\
& \times\left(\int c_{j}(t-s, y) h(j, g(j, y, t-s)) \mu(i, j, g(i, x, s))(d y)\right) d s, \\
A_{3}= & \sum_{j} \sum_{p \geq 2} \sum_{j_{1}, \ldots, j_{p-1}} \int \cdots \int_{\left\{s_{1} \geq 0, \ldots, s_{p} \geq 0, s_{1}+\cdots+s_{p} \leq t\right\}} d s_{1} \cdots d s_{p} \\
& \times c_{i}\left(s_{1}, x\right) a\left(i, j_{1}, g(i, x, s)\right) \int \mu\left(i, j_{1}, g\left(i, x, s_{1}\right)\right)\left(d y_{1}\right) \\
& \times \prod_{i=1}^{p-2} c_{j_{i}}\left(s_{i+1}, y_{i}\right) a\left(j_{i}, j_{i+1}, g\left(j_{i}, y_{i}, s_{i+1}\right)\right) \int \mu\left(j_{i}, j_{i+1}, g\left(j_{i}, y_{i}, s_{i+1}\right)\right)\left(d y_{i+1}\right) \\
& \times c_{j_{p-1}}\left(s_{p}, y_{p-1}\right) a\left(j_{p-1}, j, g\left(j_{p-1}, y_{p-1}, s_{p}\right)\right) \int \mu\left(j_{p-1}, j, g\left(j_{p-1}, y_{p-1}, s_{p}\right)\right)(d y) \\
& \times c_{j}\left(t-\left(s_{1}+\cdots+s_{p}\right), y\right) h\left(j, g\left(j, y, t-\left(s_{1}+\cdots+s_{p}\right)\right)\right) .
\end{aligned}
$$

Denoting by $M$ and $\Lambda$ respective bounds for $h$ and $\lambda$, it is easy to check that $A_{3}$ is bounded by $M \sum_{p \geq 2} \Lambda^{p} t^{p} / p$ !. Thus, $A_{3}$ divided by $t$ is uniformly bounded for $(i, x, t) \in E \times \mathbb{R}^{d} \times V$, where $V$ is a neighborhood of 0 , and tends to 0 as $t$ tends to 0 .

Under assumptions $(H), A_{2}$ divided by $t$ is bounded and tends to $\sum_{j} a(i, j, x) \int h(j$, $y) \mu(i, j, x)(d y)$ as $t$ tends to 0 .

Under assumptions $(H), A_{1}$ divided by $t$ is bounded and

$$
\frac{A_{1}}{t}=\frac{h(i, g(i, x, t))-h(i, x)}{t}-h(i, g(i, x, t)) \frac{1-c_{i}(t, x)}{t} .
$$

When $t$ tends to 0 , this quantity tends to

$$
\sum_{k=1}^{d} \frac{\partial h}{\partial x_{k}}(i, x) \mathbf{v}^{(k)}(i, x)-h(i, x) \lambda(i, x) .
$$

This completes the proof.

\section{The marginal distributions of $\left(I_{t}, X_{t}\right)$ as unique solutions of integro-differential equations}

From the previous section, we have an explicit expression for the full generator of $\left(I_{t}, X_{t}\right)_{t \geq 0}$ on regular functions (elements of $\left.D_{0}\right)$. Now, we derive a weak form of the Chapman-Kolmogorov (CK) equations with regular test functions in Section 4.1. Existence of a solution for such CK equations is clearly ensured by the construction of the 
process $\left(I_{t}, X_{t}\right)_{t \geq 0}$ of the previous section. The aim of this section then is to prove the uniqueness of the solution. Actually, the set $D_{0}$ of test functions for the CK equations (as provided by the previous section) soon appears as not rich enough to show such uniqueness. We then extend $D_{0}$ to a set of regular time-dependent functions in Section 4.2 and to less regular time-dependent functions in Section 4.3. The final conclusion is made in Section 4.4.

In this section, symbol $\rho_{0}$ stands for the initial distribution of $\left(I_{t}, X_{t}\right)_{t \geq 0}$ (with $\rho_{0} \in$ $\left.\mathscr{P}\left(E \times \mathbb{R}^{d}\right)\right)$.

4.1. The Chapman-Kolmogorov equations. We give here the expression of the CK equations for $h$ in $D_{0}$, which is a direct consequence from the expression for the full generator given in Proposition 3.5.

Proposition 4.1 (Chapman-Kolmogorov equations). Let $\rho_{t}(i, d x)$ be the marginal distribution of $\left(I_{t}, X_{t}\right)_{t \geq 0}$ at time $t$, namely, the measure on $E \times \mathbb{R}^{d}$ such that

$$
\rho_{t}(i, d x)=\sum_{i_{0} \in E} \int_{\mathbb{R}^{d}} P_{t}\left(i_{0}, x_{0}, i, d x\right) \rho_{0}\left(i_{0}, d x_{0}\right), \quad \forall t \geq 0 .
$$

Then, for all $t \in \mathbb{R}_{+}$and $h \in D_{0}$,

$$
\begin{gathered}
\sum_{i \in E} \int_{\mathbb{R}^{d}} h(i, x) \rho_{t}(i, d x)-\sum_{i \in E} \int_{\mathbb{R}^{d}} h(i, x) \rho_{0}(i, d x) \\
=\int_{0}^{t} \sum_{i \in E} \int_{\mathbb{R}^{d}}\left[\sum_{j \in E} a(i, j, x)\left(\int_{\mathbb{R}^{d}} h(j, y) \mu(i, j, x)(d y)-h(i, x)\right)\right. \\
+\mathbf{v}(i, x) \cdot \nabla h(i, x)] \rho_{s}(i, d x) d s .
\end{gathered}
$$

Such a proposition suggests the following definition.

Definition 4.2 (measure solution to CK equations). Let $\mathbf{P}: \mathbb{R}_{+} \rightarrow \mathscr{P}\left(E \times \mathbb{R}^{d}\right)$ be such that for all $t \in \mathbb{R}_{+}$and $h \in D_{0}$,

$$
\begin{gathered}
\sum_{i \in E} \int h(i, x) \mathbf{P}_{t}(i, d x)-\sum_{i \in E} \int h(i, x) \rho_{0}(i, d x) \\
=\int_{0}^{t} \sum_{i \in E} \int\left[\sum_{j \in E} a(i, j, x)\left(\int h(j, y) \mu(i, j, x)(d y)-h(i, x)\right)\right. \\
+\mathbf{v}(i, x) \cdot \nabla h(i, x)] \mathbf{P}_{s}(i, d x) d s .
\end{gathered}
$$

Then, $\mathbf{P}$ is called a measure solution of the Chapman-Kolmogorov equations.

Remark 4.3. If $\mathbf{P}$ is a measure solution of the CK equations, then $\mathbf{P}_{0}=\rho_{0}$. Also, for all $h \in C_{b}\left(\mathbb{R}^{d}, \mathbb{R}\right)^{E}$, one may check that $\sum_{i \in E} \int h(i, x) \mathbf{P}_{t}(i, d x)$ is continuous in $t$. 
Due to Proposition 4.1, we already know that a measure solution of the CK equations exists (namely, $\mathbf{P}_{t}(i, d x)=\rho_{t}(i, d x)$ ), so our problem now reduces to show the uniqueness of such a solution. If we assume that there are two measure solutions of the CK equations, we have to show that their difference, say $\widetilde{\mathbf{P}}_{s}(i, d x)$, is zero, or equivalently that

$$
\sum_{i \in E} \int_{\mathbb{R}^{d}} h(i, x) \widetilde{\mathbf{P}}_{s}(i, d x)=0,
$$

for $h$ belonging to sufficiently large set of functions, for almost all $s>0$. Alternatively, we may also prove that

$$
\sum_{i \in E} \iint_{\mathbb{R}^{d} \times \mathbb{R}_{+}} \varphi(i, x, s) \widetilde{\mathbf{P}}_{s}(i, d x) d s=0,
$$

for $\varphi$ belonging to sufficiently large set of functions. The form of (4.3) suggests the second form to be more appropriate to our problem. This leads us to extend (4.3) to some timedependent functions $\varphi$. Also, such functions will be taken with a compact support so that the term $\sum_{i \in E} \int \varphi(i, x, t) \tilde{\mathbf{P}}_{t}(i, d x)$ in (4.3) will vanish as $t \rightarrow \infty$.

\subsection{Characterization of a measure solution of the CK equations with time-dependent}

functions. The extension of (4.3) to time-dependent functions might be derived from the following equivalence (see, e.g., [11]):

$$
\begin{aligned}
& \left(\forall h \in D_{0}, h\left(I_{t}, X_{t}\right)-h\left(I_{0}, X_{0}\right)-\int_{0}^{t} \mathscr{L} h\left(I_{s}, X_{s}\right) d s \text { is a martingale }\right) \\
& \Longleftrightarrow \\
& \left(\forall \varphi \in \hat{D}_{0}, \varphi\left(I_{t}, X_{t}, t\right)-\varphi\left(I_{0}, X_{0}, 0\right)-\int_{0}^{t}\left(\frac{\partial \varphi}{\partial u}+\mathscr{L} \varphi\right)\left(I_{u}, X_{u}, u\right) d u \text { is a martingale }\right),
\end{aligned}
$$

where the new set $\hat{D}_{0}$ consists of the real continuous functions $\varphi \in C_{b}^{1}\left(\mathbb{R}^{d} \times \mathbb{R}_{+}, \mathbb{R}\right)^{E}$ such that for each $t \geq 0$, the function $\varphi_{t}:(i, x) \rightarrow \varphi(i, x, t)$ belongs to $D_{0}$. For $\varphi \in \hat{D}_{0}, \mathscr{L} \varphi$ is then defined by $\mathscr{L} \varphi(i, x, t)=\mathscr{L} \varphi_{t}(i, x)$.

However, it is better to follow here another approach, more in the spirit of the future developments, so that we now extend the CK equations directly from (4.3).

In the following, we use the symbol $\partial_{t}$ to denote the derivative with respect to the variable $t \in \mathbb{R}_{+}$, whereas we recall that $\nabla$ stands for the gradient with respect to the variable $x \in \mathbb{R}^{d}$. 
Proposition 4.4. Let $\mathbf{P}: \mathbb{R}_{+} \rightarrow \mathscr{P}\left(E \times \mathbb{R}^{d}\right)$. The function $\mathbf{P}$ is a measure solution of the Chapman-Kolmogorov equations in the sense of Definition 4.2 if and only if

$$
\begin{gathered}
\int_{0}^{t} \sum_{i \in E} \int\left[\sum_{j \in E} a(i, j, x)\left(\int \varphi(j, y, s) \mu(i, j, x)(d y)-\varphi(i, x, s)\right)+\partial_{t} \varphi(i, x, s)\right. \\
+\mathbf{v}(i, x) \cdot \nabla \varphi(i, x, s)] \mathbf{P}_{s}(i, d x) d s \\
+\sum_{i \in E}\left(\int \varphi(i, x, 0) \rho_{0}(i, d x)-\int \varphi(i, x, t) \mathbf{P}_{t}(i, d x)\right)=0,
\end{gathered}
$$

for all $t \in \mathbb{R}_{+}$and $\varphi \in C_{c}^{1}\left(\mathbb{R}^{d} \times \mathbb{R}_{+}, \mathbb{R}\right)^{E}$.

Proof. We only have to prove that (4.3) implies (4.7). The implication (4.7) $\Rightarrow(4.3)$ is clear.

For $\varphi \in C_{c}^{1}\left(\mathbb{R}^{d} \times \mathbb{R}_{+}, \mathbb{R}\right)^{E}$ and $t \in \mathbb{R}_{+}$, we first apply formula (4.3) to the function $(i, x) \rightarrow \partial_{t} \varphi(i, x, t)$. Then, we integrate such a formula with respect to $t \in(0, T)$, and we perform different integrations by parts. We use again formula (4.3) for the function $\varphi(\cdot, \cdot, T)$ and $t=T$. Thus we arrive at the desired result.

Taking $t \rightarrow \infty$ in (4.7), we now get the following result.

Corollary 4.5. If $\mathbf{P}: \mathbb{R}_{+} \rightarrow \mathscr{P}\left(E \times \mathbb{R}^{d}\right)$ is a measure solution of the $C K$ equations and $\varphi \in C_{c}^{1}\left(\mathbb{R}^{d} \times \mathbb{R}_{+}, \mathbb{R}\right)^{E}$, then the measure $m(i, d x, d s)=\mathbf{P}_{s}(i, d x) d$ s satisfies

$$
\begin{gathered}
m\left(\cdot, \mathbb{R}^{d} \times[0, T]\right)<\infty, \quad \forall T \in \mathbb{R}_{+}, \\
0=\int_{\mathbb{R}_{+}} \sum_{i \in E} \int \sum_{j \in E} a(i, j, x)\left(\int \varphi(j, y, s) \mu(i, j, x)(d y)-\varphi(i, x, s)\right) m(i, d x, d s) \\
+\int_{\mathbb{R}_{+}} \sum_{i \in E} \int\left(\partial_{t} \varphi(i, x, s)+\mathbf{v}(i, x) \cdot \nabla \varphi(i, x, s)\right) m(i, d x, d s)+\sum_{i \in E} \int \varphi(i, x, 0) \rho_{0}(i, d x) .
\end{gathered}
$$

In order to prove uniqueness of such an $m$, we assume that $\tilde{m}$ stands for the difference between two solutions. We already know

$$
\sum_{i \in E} \iint_{\mathbb{R}^{d} \times \mathbb{R}_{+}} \psi(i, x, s) \tilde{m}(i, d x, d s)=0
$$

with

$$
\begin{aligned}
\psi(i, x, s)= & \sum_{j \in E} a(i, j, x)\left(\int \varphi(j, y, s) \mu(i, j, x)(d y)-\varphi(i, x, s)\right) \\
& +\partial_{t} \varphi(i, x, s)+\mathbf{v}(i, x) \cdot \nabla \varphi(i, x, s)
\end{aligned}
$$

and $\varphi \in C_{c}^{1}\left(\mathbb{R}^{d} \times \mathbb{R}_{+}, \mathbb{R}\right)^{E}$.

To show that $\tilde{m} \equiv 0$, we still have to extend the CK equations to less regular functions $\varphi$ in order to prove that (4.9) is true for $\psi$ belonging to sufficiently large set of functions, such as $C_{c}^{1}\left(\mathbb{R}^{d} \times \mathbb{R}_{+}, \mathbb{R}\right)^{E}$. This is done in the following section. 
4.3. Extension of the time-dependent test-functions set for the CK equations. We first introduce the following definitions.

Definition 4.6. For $\varphi \in C_{b}\left(\mathbb{R}^{d} \times \mathbb{R}_{+}, \mathbb{R}\right)^{E}$, let us define $\tilde{\varphi} \in C_{b}\left(\mathbb{R}^{d} \times \mathbb{R}_{+}, \mathbb{R}\right)^{E}$ by

$$
\tilde{\varphi}(i, x, t)=\varphi(i, g(i, x, t), t)
$$

We denote by $C_{b}(d, E, \mathbf{v})$ the set of functions $\varphi \in C_{b}\left(\mathbb{R}^{d} \times \mathbb{R}_{+}, \mathbb{R}\right)^{E}$ such that the function $\tilde{\varphi}$ is continuously differentiable in $t$ with derivative $\partial_{t} \tilde{\varphi} \in C_{b}\left(\mathbb{R}^{d} \times \mathbb{R}_{+}, \mathbb{R}\right)^{E}$.

Remark 4.7. Note that, thanks to Lemma 2.1, the functions $\varphi$ and the functions $\tilde{\varphi}$ are in one-to-one correspondence because

$$
\varphi(i, x, t)=\tilde{\varphi}(i, g(i, x,-t), t)
$$

If $\tilde{\varphi}$ is continuously differentiable in $t$ and such that its derivative $\partial_{t} \tilde{\varphi} \in C_{b}\left(\mathbb{R}^{d} \times \mathbb{R}_{+}, \mathbb{R}\right)^{E}$, then the function $\varphi$ defined by (4.12) belongs to $C_{b}(d, E, \mathbf{v})$. Also, note that if $\varphi \in C_{b}^{1}\left(\mathbb{R}^{d} \times\right.$ $\left.\mathbb{R}_{+}, \mathbb{R}\right)^{E}$, the function $\partial_{t} \varphi$ belongs to $C_{b}\left(\mathbb{R}^{d} \times \mathbb{R}_{+}, \mathbb{R}\right)^{E}$ and is such that

$$
\partial_{t} \tilde{\varphi}(i, y, s)=\partial_{t} \varphi(i, g(i, y, s), s)+\mathbf{v}(i, g(i, y, s)) \cdot \nabla \varphi(i, g(i, y, s), s) .
$$

This yields

$$
\partial_{t} \tilde{\varphi}(i, g(i, x,-s), s)=\partial_{t} \varphi(i, x, s)+\mathbf{v}(i, x) \cdot \nabla \varphi(i, x, s), \quad \forall(i, x, s) \in E \times \mathbb{R}^{d} \times \mathbb{R}_{+},
$$

which is the usual expression of a partial derivative of the velocity field $\mathbf{v}(i, \cdot)$.

This last expression leads to the following notation: for all $\varphi \in C_{b}(d, E, \mathbf{v})$, we denote by $\partial_{t, \mathbf{v}} \varphi \in C_{b}\left(\mathbb{R}^{d} \times \mathbb{R}_{+}, \mathbb{R}\right)^{E}$ the function defined by

$$
\partial_{t, \mathbf{v}} \varphi(i, x, s)=\partial_{t} \tilde{\varphi}(i, g(i, x,-s), s)
$$

(Recall that $\partial_{t} \tilde{\varphi}$ is the partial derivative of $\tilde{\varphi}$ with respect to its third argument.)

Remark 4.8. Suppose that $\varphi \in C_{b}^{1}\left(\mathbb{R}^{d} \times \mathbb{R}_{+}, \mathbb{R}\right)^{E}$ with $\partial_{t} \varphi(i, \cdot, \cdot)+\mathbf{v}(i, \cdot) \cdot \nabla \varphi(i, \cdot, \cdot) \in$ $C_{b}\left(\mathbb{R}^{d} \times \mathbb{R}_{+}, \mathbb{R}\right)^{E}$. Then, $\varphi \in C_{b}(d, E, \mathbf{v})$. In particular, any function $\varphi \in C_{c}^{1}\left(\mathbb{R}^{d} \times \mathbb{R}_{+}, \mathbb{R}\right)^{E}$ belongs to $C_{b}(d, E, \mathbf{v})$. Similarly, if we take any function $\varphi \in C_{b}^{1}\left(\mathbb{R}^{d}, \mathbb{R}\right)^{E}$ which is constant in time and such that $\mathbf{v}(i, \cdot) \cdot \nabla \varphi(i, \cdot) \in C_{b}\left(\mathbb{R}^{d}, \mathbb{R}\right)$, then $\varphi \in C_{b}(d, E, \mathbf{v})$.

We will also need the following result.

Lemma 4.9. If $\tilde{\varphi} \in C_{c}^{1}\left(\mathbb{R}^{d} \times \mathbb{R}_{+}, \mathbb{R}\right)^{E}$, then $\varphi \in C_{c}^{1}\left(\mathbb{R}^{d} \times \mathbb{R}_{+}, \mathbb{R}\right)^{E}$.

Proof. Let $\tilde{\varphi} \in C_{c}^{1}\left(\mathbb{R}^{d} \times \mathbb{R}_{+}, \mathbb{R}\right)^{E}$. The only point to show is that the function $\varphi$ has a compact support: as $\tilde{\varphi}$ has a compact support, there are some constants $M>0, T>0$ such that $\tilde{\varphi}(i, x, t)=0$ if $t>T$ or $\|x\|>M$, for all $i \in E$. Now, let $(i, x, t)$ be such that $\varphi(i, x, t) \neq 0$. 
We derive that $0 \leq t \leq T$ and $\|g(i, x,-t)\| \leq M$. Then,

$$
\begin{aligned}
\|x\| & \leq\|x-g(i, x,-t)\|+\|g(i, x,-t)\| \\
& \leq\|g(i, g(i, x,-t), t)-g(i, g(i, x,-t), 0)\|+M \\
& \leq \int_{0}^{t}\|\mathbf{v}(i, g(i, g(i, x,-t), s))\| d s+M \\
& \leq V_{1} \int_{0}^{t}\|g(i, g(i, x,-t), s)\| d s+V_{2} t+M .
\end{aligned}
$$

Moreover, for $0 \leq s \leq t \leq T$, we have

$$
\begin{aligned}
\|g(i, g(i, x,-t), s)\| & \leq\|g(i, x,-t)\|+\|g(i, g(i, x,-t), s)-g(i, g(i, x,-t), 0)\| \\
& \leq M+V_{1} \int_{0}^{s}\|g(i, g(i, x,-t), u)\| d u+V_{2} s \quad \text { (as previously) } \\
& \leq M+V_{2} T+V_{1} \int_{0}^{s}\|g(i, g(i, x,-t), u)\| d u .
\end{aligned}
$$

Using Gronwall's lemma, we derive

$$
\|g(i, g(i, x,-t), s)\| \leq\left(M+V_{2} T\right) e^{V_{1} s} \leq\left(M+V_{2} T\right) e^{V_{1} T} .
$$

Inequality (4.16) now implies that

$$
\|x\| \leq V_{1} T\left(M+V_{2} T\right) e^{V_{1} T}+V_{2} T+M,
$$

and hence $\varphi$ has a compact support. This completes the proof.

We are now ready to state the main result of this section.

Proposition 4.10. Let $m$ be a Radon measure such that $m\left(\cdot, \mathbb{R}^{d} \times[0, T]\right)<\infty$ for all $T \in$ $\mathbb{R}_{+}$, and (4.8) are fulfilled for all $\varphi \in C_{c}^{1}\left(\mathbb{R}^{d} \times \mathbb{R}_{+}, \mathbb{R}\right)^{E}$. Then,

$$
\begin{aligned}
0= & \int_{\mathbb{R}_{+}} \sum_{i \in E} \int \sum_{j \in E} a(i, j, x)\left(\int \varphi(j, y, s) \mu(i, j, x)(d y)-\varphi(i, x, s)\right) m(i, d x, d s) \\
& +\int_{\mathbb{R}_{+}} \sum_{i \in E} \int \partial_{t, \mathbf{v}} \varphi(i, x, s) m(i, d x, d s)+\sum_{i \in E} \int \varphi(i, x, 0) \rho_{0}(i, d x),
\end{aligned}
$$

for all functions $\varphi \in C_{b}(d, E, \mathbf{v})$ such that $\varphi(\cdot, \cdot, t)=0$ for all $t>T$, for some $T>0$.

Proof. We first prove the result in the case $\varphi \in C_{b}(d, E, \mathbf{v})$ is Lipschitz continuous with a compact support.

We need classical mollifying functions. First, let $C_{c}^{\infty}(\mathbb{R})$ (resp., $C_{c}^{\infty}\left(\mathbb{R}^{d}\right)$ ) be the set of real-valued infinitely differentiable functions with a compact support in $\mathbb{R}$ (resp., $\mathbb{R}^{d}$ ). Then, let $\bar{\rho}_{1} \in C_{c}^{\infty}(\mathbb{R})$ be such that $\bar{\rho}_{1}(s)=0$ for all $s \in(-\infty,-1] \cup[0,+\infty), \bar{\rho}_{1}(s) \geq 0$ for all $s \in[-1,0]$ and $\int_{\mathbb{R}} \bar{\rho}_{1}(s) d s=1$. For all $n \in \mathbb{N}^{*}$, we define the function $\bar{\rho}_{n} \in C_{c}^{\infty}(\mathbb{R})$ such that $\bar{\rho}_{n}(s)=n \bar{\rho}_{1}(n s)$, for all $s \in \mathbb{R}$. We also introduce a function $\rho_{1} \in C_{c}^{\infty}\left(\mathbb{R}^{d}\right)$ such that $\rho_{1}(x)=0$ for all $x \in \mathbb{R}^{d}$ with $|x| \geq 1, \rho_{1}(x) \geq 0$ for all $x \in \mathbb{R}^{d}$ and $\int_{\mathbb{R}^{d}} \rho_{1}(x) d x=1$. For all $n \in \mathbb{N}^{*}$, we then define the function $\rho_{n} \in C_{c}^{\infty}\left(\mathbb{R}^{d}\right)$ by $\rho_{n}(x)=n^{d} \rho_{1}(n x)$, for $x \in \mathbb{R}^{d}$. 
Now, let $\varphi \in C_{b}(d, E, \mathbf{v})$, and $\varphi$ is assumed to be Lipschitz continuous with compact support. We define, for $n \in \mathbb{N}^{*}, \tilde{x} \in \mathbb{R}^{d}$, and $\tilde{t} \in \mathbb{R}$, the function $\varphi_{n}(\tilde{x}, \tilde{t}, \cdot, \cdot, \cdot)$ by

$$
\varphi_{n}(\tilde{x}, \tilde{t}, i, x, t)=\varphi(i, \tilde{x}, \tilde{t}) \rho_{n}(x-\tilde{x}) \bar{\rho}_{n}(t-\tilde{t}), \quad \forall(x, t) \in \mathbb{R}^{d} \times \mathbb{R}_{+} .
$$

Since $\varphi_{n}(\tilde{x}, \tilde{t}, \cdot, \cdot, \cdot) \in C_{c}^{1}\left(\mathbb{R}^{d} \times \mathbb{R}_{+}, \mathbb{R}\right)^{E}$, we may now use the function $\varphi_{n}(\tilde{x}, \tilde{t}, \cdot, \cdot \cdot \cdot \cdot)$ in (4.8). Integrating on $\mathbb{R}_{+} \times \mathbb{R}^{d}$, we get $A_{1, n}+A_{2, n}+A_{3, n}+A_{4, n}=0$, where

$$
\begin{aligned}
& A_{1, n}=\iint_{\mathbb{R}^{d} \times \mathbb{R}_{+}} \int_{\mathbb{R}_{+}} \sum_{i \in E} \int \sum_{j \in E} a(i, j, x) \int \varphi_{n}(\tilde{x}, \tilde{t}, j, y, s) \mu(i, j, x)(d y) m(i, d x, d s) d \tilde{x} d \tilde{t}, \\
& A_{2, n}=-\iint_{\mathbb{R}^{d} \times \mathbb{R}_{+}} \int_{\mathbb{R}_{+}} \sum_{i \in E} \int \sum_{j \in E} a(i, j, x) \varphi_{n}(\tilde{x}, \tilde{t}, i, x, s) m(i, d x, d s) d \tilde{x} d \tilde{t}, \\
& A_{3, n}=\iint_{\mathbb{R}^{d} \times \mathbb{R}_{+}} \int_{\mathbb{R}_{+}} \sum_{i \in E} \int\left(\partial_{t} \varphi_{n}(\tilde{x}, \tilde{t}, i, x, s)+\mathbf{v}(i, x) \cdot \nabla_{x} \varphi_{n}(\tilde{x}, \tilde{t}, i, x, s)\right) m(i, d x, d s) d \tilde{x} d \tilde{t}, \\
& A_{4, n}=\iint_{\mathbb{R}^{d} \times \mathbb{R}_{+}} \sum_{i \in E} \int \varphi_{n}(\tilde{x}, \tilde{t}, i, x, 0) \rho_{0}(i, d x) d \tilde{x} d \tilde{t} .
\end{aligned}
$$

The notation $\nabla_{x}$ in $A_{3, n}$ is the gradient with respect to $x$.

We now study the limit of $A_{i, n}$ as $n \rightarrow \infty$ for $i=1,2,3,4$. The continuity of $\varphi$ implies that

$$
\lim _{n \rightarrow \infty} \iint_{\mathbb{R}^{d} \times \mathbb{R}_{+}} \varphi_{n}(\tilde{x}, \tilde{t}, i, x, t) d \tilde{x} d \tilde{t}=\varphi(i, x, t), \quad \forall(x, t) \in \mathbb{R}^{d} \times \mathbb{R}_{+} .
$$

From Fubini's theorem and the dominated convergence theorem, we derive that

$$
\lim _{n \rightarrow \infty} \iint_{\mathbb{R}^{d} \times \mathbb{R}_{+}} \int \varphi_{n}(\tilde{x}, \tilde{t}, j, y, s) \mu(i, j, x)(d y) d \tilde{x} d \tilde{t}=\int \varphi(j, y, s) \mu(i, j, x)(d y),
$$

for all $i, j \in E$ and $(x, s) \in \mathbb{R}^{d} \times \mathbb{R}_{+}$. Moreover, we have

$$
\lim _{n \rightarrow \infty} A_{1, n}=\int_{\mathbb{R}_{+}} \sum_{i \in E} \int \sum_{j \in E} a(i, j, x) \int \varphi(j, y, s) \mu(i, j, x)(d y) m(i, d x, d s),
$$

and similarly

$$
\lim _{n \rightarrow \infty} A_{2, n}=-\int_{\mathbb{R}_{+}} \sum_{i \in E} \int \sum_{j \in E} a(i, j, x) \varphi(i, x, s) m(i, d x, d s) .
$$

We now turn to the study of $A_{3, n}$, and we define

$$
B_{n}(i, x, t)=\iint_{\mathbb{R}^{d} \times \mathbb{R}_{+}}\left(\partial_{t} \varphi_{n}(\tilde{x}, \tilde{t}, i, x, t)+\mathbf{v}(i, x) \cdot \nabla_{x} \varphi_{n}(\tilde{x}, \tilde{t}, i, x, t)\right) d \tilde{x} d \tilde{t}
$$


Using the property $\partial_{t} \bar{\rho}_{n}(t-\tilde{t})=-\partial_{\tilde{t}} \bar{\rho}_{n}(t-\tilde{t})$ and $\nabla_{x} \rho_{n}(x-\tilde{x})=-\nabla_{\tilde{x}} \rho_{n}(x-\tilde{x})$, and thanks to the fact that $\varphi$ is Lipschitz continuous with compact support, we can perform an integration by parts (because $\varphi$ is continuous with bounded variation). Noticing that the integrated terms in time vanish since $\varphi(i, \tilde{x}, \tilde{t}) \bar{\rho}_{n}(t-\tilde{t})=0$ for $\tilde{t}>t+1$ and $\tilde{t}=0$ in view of the definition of $\bar{\rho}_{1}$, we get $B_{n}(i, x, t)=B_{1, n}(i, x, t)+B_{2, n}(i, x, t)$, where

$$
\begin{aligned}
B_{1, n}(i, x, t) & =\iint_{\mathbb{R}^{d} \times \mathbb{R}_{+}}\left(\partial_{t} \varphi(i, \tilde{x}, \tilde{t})+\mathbf{v}(i, \tilde{x}) \cdot \nabla \varphi(i, \tilde{x}, \tilde{t})\right) \bar{\rho}_{n}(t-\tilde{t}) \rho_{n}(x-\tilde{x}) d \tilde{x} d \tilde{t} \\
& =\iint_{\mathbb{R}^{d} \times \mathbb{R}_{+}} \partial_{t, \mathbf{v}} \varphi(i, \tilde{x}, \tilde{t}) \bar{\rho}_{n}(t-\tilde{t}) \rho_{n}(x-\tilde{x}) d \tilde{x} d \tilde{t}, \\
B_{2, n}(i, x, t) & =\iint_{\mathbb{R}^{d} \times \mathbb{R}_{+}}(\mathbf{v}(i, x)-\mathbf{v}(i, \tilde{x})) \cdot \nabla \varphi(i, \tilde{x}, \tilde{t}) \bar{\rho}_{n}(t-\tilde{t}) \rho_{n}(x-\tilde{x}) d \tilde{x} d \tilde{t}
\end{aligned}
$$

For $B_{1, n}$, the assumption $\varphi \in C_{b}(d, E, \mathbf{v})$ implies that $B_{1, n}(i, x, t) \rightarrow \partial_{t, \mathbf{v}} \varphi(i, x, t)$ as $n \rightarrow+\infty$ for all $(x, t) \in \mathbb{R}^{d} \times \mathbb{R}_{+}$.

For $B_{2, n}$, we note that, if $\nabla \varphi(i, \tilde{x}, \tilde{t}) \rho_{n}(x-\tilde{x}) \neq 0$, then $\|x-\tilde{x}\| \leq 1 / n \leq 1$ and $\tilde{x} \in K$, a compact set. Hence, $B_{2, n}(i, x, t)=0$ if $x \notin \cup_{\tilde{x} \in K} B(\tilde{x}, 1)$, where $B(\tilde{x}, 1)$ is the ball centered on $\tilde{x}$ with radius 1 . Besides, as $\mathbf{v}$ is locally Lipschitz continuous, we may use its Lipschitz constant $C_{\mathbf{v}}$ on $\cup_{\tilde{x} \in K} B(\tilde{x}, 1)$. We get $|\mathbf{v}(i, x)-\mathbf{v}(i, \tilde{x})| \leq C_{\mathbf{v}} / n$ if $x \in \cup_{\tilde{x} \in K} B(\tilde{x}, 1)$ and

$$
\left|B_{2, n}(i, x, t)\right| \leq \frac{C_{\mathbf{v}}}{n}\|\nabla \varphi\|_{\infty} .
$$

This proves that $B_{2, n}(i, x, t) \rightarrow 0$ as $n \rightarrow+\infty$ for all $(x, t) \in \mathbb{R}^{d} \times \mathbb{R}_{+}$.

We now derive that, for all $(x, s) \in \mathbb{R}^{d} \times \mathbb{R}_{+}$, we have

$$
\lim _{n \rightarrow \infty} B_{n}(i, x, s)=\partial_{t, \mathbf{v}} \varphi(i, x, s) .
$$

Since $\mathbf{v}$ is bounded on a compact support, $\left|\partial_{t} \varphi(i, \tilde{x}, \tilde{t})+\mathbf{v}(i, x) \cdot \nabla \varphi(i, \tilde{x}, \tilde{t})\right|$ is bounded by some constant $M>0$ and consequently

$$
\left|B_{n}(i, x, t)\right|=\left|\iint_{\mathbb{R}^{d} \times \mathbb{R}_{+}}\left(\partial_{t} \varphi(i, \tilde{x}, \tilde{t})+\mathbf{v}(i, x) \cdot \nabla \varphi(i, \tilde{x}, \tilde{t})\right) \bar{\rho}_{n}(t-\tilde{t}) \rho_{n}(x-\tilde{x}) d \tilde{x} d \tilde{t}\right| \leq M .
$$

Now, using (4.30), Fubini's theorem and the dominated convergence theorem, we conclude that

$$
\lim _{n \rightarrow \infty} A_{3, n}=\int_{\mathbb{R}_{+}} \sum_{i \in E} \int \partial_{t, \mathbf{v}} \varphi(i, x, s) m(i, d x, d s) .
$$

Finally, for $A_{4, n}$, by using (4.23), we find that

$$
\lim _{n \rightarrow \infty} \iint_{\mathbb{R}^{d} \times \mathbb{R}_{+}} \varphi(i, \tilde{x}, \tilde{t}) \rho_{n}(x-\tilde{x}) \bar{\rho}_{n}(0-\tilde{t}) d \tilde{x} d \tilde{t}=\varphi(i, x, 0), \quad \forall x \in \mathbb{R}^{d} .
$$


Once again, Fubini's theorem and the dominated convergence theorem imply that

$$
\lim _{n \rightarrow \infty} A_{4, n}=\sum_{i \in E} \int \varphi(i, x, 0) \rho_{0}(i, d x)
$$

Gathering together (4.25), (4.26), (4.32), and (4.34) yield (4.20) for all function $\varphi \in$ $C_{b}(d, E, \mathbf{v})$ which is Lipschitz continuous with a compact support.

Now, let us assume that $\varphi \in C_{b}(d, E, \mathbf{v})$ is such that there exists $T_{0}>0$ with $\varphi(\cdot, \cdot, t)=0$ for all $t>T_{0}$.

We take a function $H_{1} \in C_{c}^{\infty}(\mathbb{R})$ such that $H_{1}(x)=1$ for $x \in[-1,1], H_{1}(x) \in[0,1]$ for $x \in \mathbb{R}$, and $H_{1}(x)=0$ for $x \in(-\infty,-2] \cup[2,+\infty)$. We also define, for $n \in \mathbb{N}^{*}$ and $(i, y, t) \in E \times \mathbb{R}^{d} \times \mathbb{R}_{+}$,

$$
\tilde{\varphi}_{n}(i, y, t)=H_{1}\left(\frac{y^{2}}{n^{2}}\right) \int_{\mathbb{R}^{d}} \tilde{\varphi}(i, \tilde{y}, t) \rho_{n}(y-\tilde{y}) d \tilde{y}=H_{1}\left(\frac{y^{2}}{n^{2}}\right) \int_{\mathbb{R}^{d}} \varphi(i, g(i, \tilde{y}, t), t) \rho_{n}(y-\tilde{y}) d \tilde{y} .
$$

Also, let

$$
\varphi_{n}(i, x, t)=\tilde{\varphi}_{n}(i, g(i, x,-t), t), \quad(i, x, t) \in E \times \mathbb{R}^{d} \times \mathbb{R}_{+} .
$$

We now have $\tilde{\varphi}_{n} \in C_{c}^{1}\left(\mathbb{R}^{d} \times \mathbb{R}_{+}\right)^{E}$ and $\varphi_{n} \in C_{b}(d, E, \mathbf{v})$. Moreover, Lemma 4.9 implies that $\varphi_{n}$ has a compact support. As $\varphi_{n} \in C_{b}(d, E, \mathbf{v})$ is locally Lipschitz continuous with compact support, it is Lipschitz continuous and we may apply (4.20) to $\varphi_{n}$. Also, since $H_{1}\left(y^{2} / n^{2}\right)=1$ for $y \in \mathbb{R}^{d}$ and for $n$ large enough, we get

$$
\lim _{n \rightarrow \infty} \tilde{\varphi}_{n}(i, y, t)=\tilde{\varphi}(i, y, t), \quad \lim _{n \rightarrow \infty} \partial_{t} \tilde{\varphi}_{n}(i, y, t)=\partial_{t} \tilde{\varphi}(i, y, t)
$$

for all $(y, t) \in \mathbb{R}^{d} \times \mathbb{R}_{+}$. This implies that, for all $(x, t) \in \mathbb{R}^{d} \times \mathbb{R}_{+}$,

$$
\lim _{n \rightarrow \infty} \varphi_{n}(i, x, t)=\varphi(i, x, t), \quad \lim _{n \rightarrow \infty} \partial_{t, \mathbf{v}} \varphi_{n}(i, x, t)=\partial_{t, \mathbf{v}} \varphi(i, x, t) .
$$

Taking $n \rightarrow \infty$ in (4.20) for $\varphi_{n}$, we now get (4.20) for $\varphi$, in view of the dominated convergence theorem (using the fact that $m\left(\cdot, \mathbb{R}^{d} \times\left[0, T_{0}\right]\right)$ is finite for all $T_{0} \in \mathbb{R}_{+}$, and that $\varphi_{n}$ and $\partial_{t, \mathbf{v}} \varphi_{n}$ remain bounded by $\|\varphi\|_{\infty} \mathbf{1}_{E \times \mathbb{R}^{d} \times\left[0, T_{0}\right]}$ and $\left\|\partial_{t, \mathbf{v}} \varphi\right\|_{\infty} \mathbf{1}_{E \times \mathbb{R}^{d} \times\left[0, T_{0}\right]}$, resp.). This concludes the proof of Proposition 4.10.

4.4. The uniqueness result. We are now ready to state the uniqueness of a Radon measure $m$, the solution to (4.8), as exposed in the following theorem and its corollary.

THEOREM 4.11. There is at most one Radon measure $m$ on $E \times \mathbb{R}^{d} \times \mathbb{R}_{+}$satisfying the condition $m\left(\cdot, \mathbb{R}^{d} \times[0, T]\right)<\infty$ for all $T \in \mathbb{R}_{+}$, and (4.8) for all $\varphi \in C_{c}^{1}\left(\mathbb{R}^{d} \times \mathbb{R}_{+}, \mathbb{R}\right)^{E}$.

Before proving the theorem, we derive the following corollary which finally proves the uniqueness of the measure solution to the CK equations. This corollary is a direct consequence from Theorem 4.11, Corollary 4.5, and the continuity property of $\mathbf{P}_{t}$ with respect to $t$ (see Remark 4.3). 
Corollary 4.12. There is at most one function $\mathbf{P}: \mathbb{R}_{+} \rightarrow \mathscr{P}\left(E \times \mathbb{R}^{d}\right)$, which is a measure solution of the CK equations in the sense of Definition 4.2.

Proof of Theorem 4.11. Suppose that there are two Radon measures $m_{1}$ and $m_{2}$ such that $m_{i}\left(\cdot, \mathbb{R}^{d} \times[0, T]\right)<\infty$ for all $T \in \mathbb{R}_{+}, i=1,2$, and which are solutions of $(4.8)$. Let $\tilde{m}$ be the difference between these two solutions. We have to prove that $\tilde{m}$ is equal to 0 . In order to do this, we are going to show that

$$
\sum_{i \in E} \iint_{\mathbb{R}^{d} \times \mathbb{R}_{+}} \psi(i, x, s) \tilde{m}(i, d x, d s)=0, \quad \forall \psi \in C_{c}^{1}\left(\mathbb{R}^{d} \times \mathbb{R}_{+}, \mathbb{R}\right)^{E} .
$$

Note that, thanks to Proposition 4.10, we already know that

$$
\sum_{i \in E} \iint_{\mathbb{R}^{d} \times \mathbb{R}_{+}}\left[\sum_{j \in E} a(i, j, x)\left(\int \varphi(j, y, s) \mu(i, j, x)(d y)-\varphi(i, x, s)\right)+\partial_{t, \mathbf{v}} \varphi(i, x, s)\right] \tilde{m}(i, d x, d s)=0,
$$

for all $\varphi \in C_{b}(d, E, \mathbf{v})$ such that there exists $T>0$ with $\varphi(\cdot, \cdot, t)=0$ for all $t \geq T$.

Now, let $\psi \in C_{c}^{1}\left(\mathbb{R}^{d} \times \mathbb{R}_{+}, \mathbb{R}\right)^{E}$. We have to show the existence of $\varphi \in C_{b}(d, E, \mathbf{v})$ such that:

(1) there exists $T>0$ with $\varphi(\cdot, \cdot, t)=0$ for all $t>T$;

(2) for all $i \in E, t \in \mathbb{R}_{+}$, and $x \in \mathbb{R}^{d}$,

$$
\psi(i, x, t)=\partial_{t, \mathbf{v}} \varphi(i, x, t)-\sum_{j \in E} a(i, j, x)\left(\int \varphi(j, y, t) \mu(i, j, x)(d y)-\varphi(i, x, t)\right) .
$$

Alternatively (see Remark 4.7), we have to show the existence of $\tilde{\varphi} \in C_{b}\left(\mathbb{R}^{d} \times \mathbb{R}_{+}, \mathbb{R}\right)^{E}$ such that, for all $i \in E, s \in \mathbb{R}_{+}$, and $\tilde{x} \in \mathbb{R}^{d}$,

$$
\begin{aligned}
\partial_{s} \tilde{\varphi}(i, \tilde{x}, s)= & \psi(i, g(i, \tilde{x}, s), s) \\
& -\sum_{j \in E} a(i, j, g(i, \tilde{x}, s))\left(\int \tilde{\varphi}(j, g(i, y,-s), s) \mu(i, j, g(i, \tilde{x}, s))(d y)-\tilde{\varphi}(i, \tilde{x}, s)\right),
\end{aligned}
$$

and also that

$$
\tilde{\varphi}(\cdot, \cdot, T)=0,
$$

where $T$ is such that $\psi(\cdot, \cdot, t)=0$ for all $t>T$.

Let $R: C_{b}\left(\mathbb{R}^{d}, \mathbb{R}\right)^{E} \times \mathbb{R}_{+} \rightarrow C_{b}\left(\mathbb{R}^{d}, \mathbb{R}\right)^{E}$ be such that, for all $h \in C_{b}\left(\mathbb{R}^{d}, \mathbb{R}\right)^{E}$,

$$
\begin{aligned}
R(h, s)(i, \tilde{x})= & \psi(i, g(i, \tilde{x}, s), s) \\
& -\sum_{j \in E} a(i, j, g(i, \tilde{x}, s))\left(\int h(j, g(i, y,-s)) \mu(i, j, g(i, \tilde{x}, s))(d y)-h(i, \tilde{x})\right) .
\end{aligned}
$$


Equation (4.42) can be written as

$$
\partial_{s} \tilde{\varphi}(i, \tilde{x}, s)=R(\tilde{\varphi}(\cdot, s), s)(\tilde{x})
$$

Moreover,

$$
\left|R(h, s)(i, \tilde{x})-R\left(h^{\prime}, s\right)(i, \tilde{x})\right| \leq 2 A \operatorname{card}(E)\left\|h-h^{\prime}\right\|_{\infty},
$$

where $A$ has been defined in assumptions $(H)$, assertion 1 . The operator $R$ is globally Lipschitz continuous with respect to its first argument, and one can also check that $R$ is continuous with respect to its second argument and that it is sublinear. Usual methods for ordinary differential equations (see, e.g., [1]) then lead to global existence and uniqueness of a solution for (4.42)-(4.43). In particular, we get the existence of a function $\tilde{\varphi} \in C_{b}\left(\mathbb{R}^{d} \times \mathbb{R}_{+}, \mathbb{R}\right)^{E}$, solution of (4.42)-(4.43). This concludes the proof.

Remark 4.13. This uniqueness result for the Chapman-Kolmogorov equation implies that, for each $\rho_{0} \in \mathscr{P}\left(E \times R^{d}\right)$, any two solutions of the martingale problem for $\left(\mathscr{L}, \rho_{0}\right)$ have the same one-dimensional distributions. Then, general results on Markov processes (see, e.g., [10, Theorem 4.2]) assert that the process $\left(I_{t}, X_{t}\right)_{t \geq 0}$ is strong Markov. This gives, in the framework of this paper, an alternative proof of the strong Markov property shown by Davis $[7,8]$ for general piecewise deterministic Markov processes.

\section{Conclusion}

In this paper, we have characterized the marginal distribution of a piecewise deterministic Markov process $\left(I_{t}, X_{t}\right)_{t \in \mathbb{R}_{+}}$as the unique solution of a set of explicit integro-differential equations. This characterization is used in another paper [4] to construct some numerical approximation of $\rho_{t}(i, d x)$, which is proved to converge weakly towards $\rho_{t}(i, d x)$. The uniqueness of the solution of the CK equations, namely, the main part of the present paper, is crucial to show the convergence of the numerical approximation towards the right distribution. Such numerical approximation has already been tested on a few analytical benchmarks, showing the accuracy of the method (see [3]).

\section{Acknowledgment}

The authors warmly thank the associate editor and referees for their thorough reading of the paper which helped a lot improving the presentation of the paper.

\section{References}

[1] H. Amann, Ordinary Differential Equations, de Gruyter Studies in Mathematics, vol. 13, Walter de Gruyter, Berlin, 1990.

[2] K. Borovkov and A. Novikov, On a piece-wise deterministic Markov process model, Statistics \& Probability Letters 53 (2001), no. 4, 421-428.

[3] C. Cocozza-Thivent, R. Eymard, and S. Mercier, A Numerical scheme to solve integro-differential equations in the dynamic reliability field, PSAM 7/ESREL '04, Special Technical Session: "Dynamic reliability: Methods and applications", Springer, Berlin, 2004, pp. 675-680.

[4] _ A numerical scheme for solving integro-differential equations from the dynamic reliability field, to appear in IMA Journal of Numerical Analysis. 
[5] O. L. V. Costa and F. Dufour, On the poisson equation for piecewise-deterministic Markov processes, SIAM Journal on Control and Optimization 42 (2003), no. 3, 985-1001.

[6] O. L. V. Costa, C. A. B. Raymundo, and F. Dufour, Optimal stopping with continuous control of piecewise deterministic Markov processes, Stochastics and Stochastic Reports 70 (2000), no. 1-2, 41-73.

[7] M. H. A. Davis, Piecewise-deterministic Markov processes: a general class of nondiffusion stochastic models, Journal of the Royal Statistical Society. Series B. Methodological 46 (1984), no. 3, 353388.

[8] - Markov Models and Optimization, Monographs on Statistics and Applied Probability, vol. 49, Chapman \& Hall, London, 1993.

[9] F. Dufour and O. L. V. Costa, Stability of piecewise-deterministic Markov processes, SIAM Journal on Control and Optimization 37 (1999), no. 5, 1483-1502.

[10] S. N. Ethier and T. G. Kurtz, Markov Processes. Characterization and Convergence, Wiley Series in Probability and Mathematical Statistics: Probability and Mathematical Statistics, John Wiley \& Sons, New York, 1986.

[11] R. A. Holley and D. W. Stroock, A martingale approach to infinite systems of interacting processes, The Annals of Probability 4 (1976), no. 2, 195-228.

[12] P. E. Labeau, Méthodes semi-analytiques et outils de simulation en dynamique probabiliste, Ph.D. thesis, Faculté des Sciences Appliquées, Service de Métrologie Nucléaire, ULB, Bruxelles, 1996.

[13] T. Rolski, H. Schmidli, V. Schmidt, and J. Teugels, Stochastic Processes for Insurance and Finance, Wiley Series in Probability and Statistics, John Wiley \& Sons, Chichester, 1999.

Christiane Cocozza-Thivent: Laboratoire d'Analyse et de Mathématiques Appliquées

(CNRS UMR 8050), Université de Marne-la-Vallée, 5, boulevard Descartes,

Champs-sur-Marne, 77454 Marne-la-Vallée cedex 2, France

E-mail address: christiane.cocozza@univ-mlv.fr

Robert Eymard: Laboratoire d'Analyse et de Mathématiques Appliquées (CNRS UMR 8050), Université de Marne-la-Vallée, 5, boulevard Descartes, Champs-sur-Marne,

77454 Marne-la-Vallée cedex 2, France

E-mail address: robert.eymard@univ-mlv.fr

Sophie Mercier: Laboratoire d'Analyse et de Mathématiques Appliquées (CNRS UMR 8050), Université de Marne-la-Vallée, 5, boulevard Descartes, Champs-sur-Marne,

77454 Marne-la-Vallée cedex 2, France

E-mail address: sophie.mercier@univ-mlv.fr

Michel Roussignol: Laboratoire d'Analyse et de Mathématiques Appliquées (CNRS UMR 8050), Université de Marne-la-Vallée, 5, boulevard Descartes, Champs-sur-Marne,

77454 Marne-la-Vallée cedex 2, France

E-mail address: michel.roussignol@univ-mlv.fr 


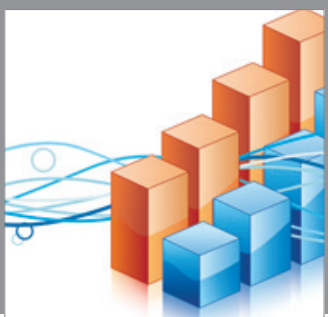

Advances in

Operations Research

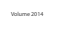

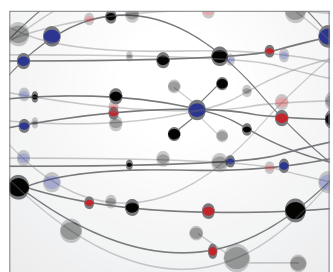

\section{The Scientific} World Journal
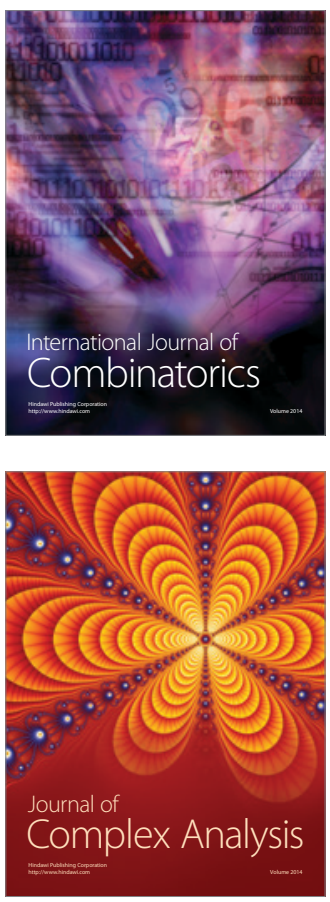

International Journal of

Mathematics and

Mathematical

Sciences
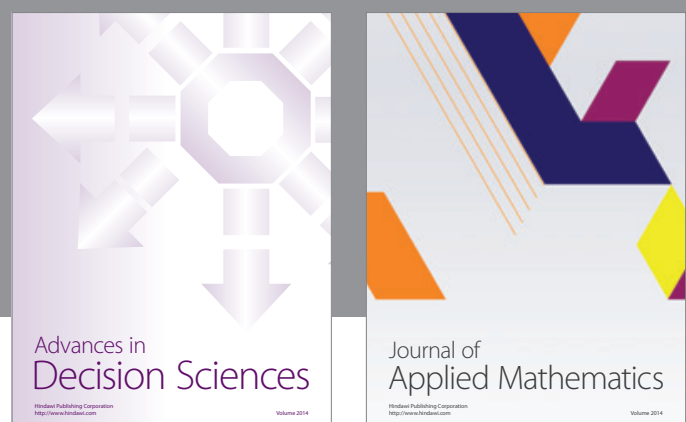

Journal of

Applied Mathematics
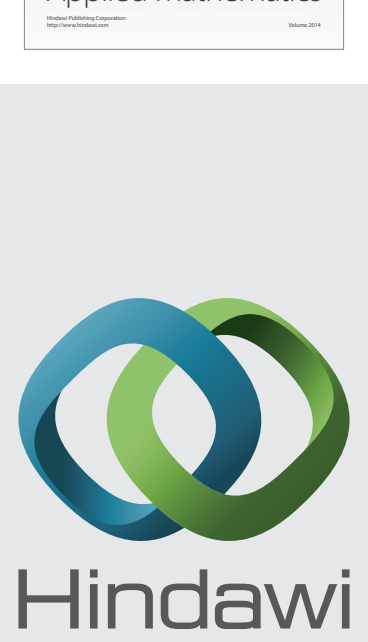

Submit your manuscripts at http://www.hindawi.com
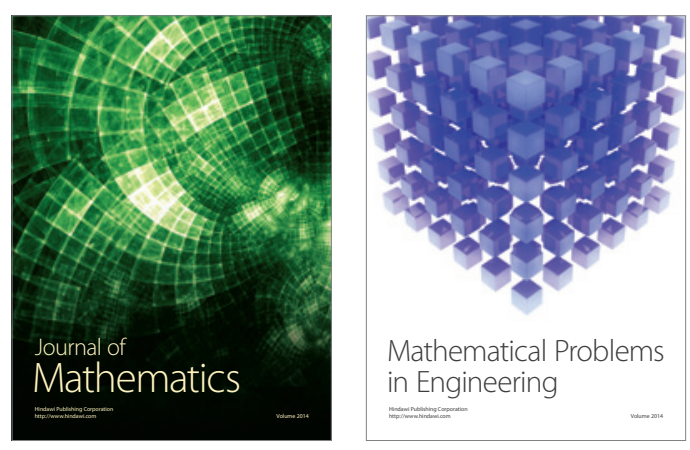

Mathematical Problems in Engineering
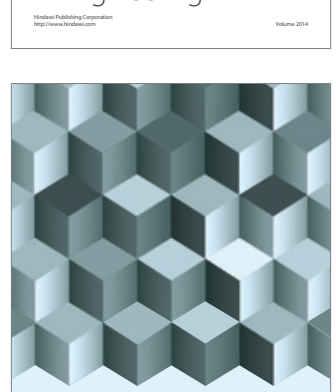

Journal of

Function Spaces
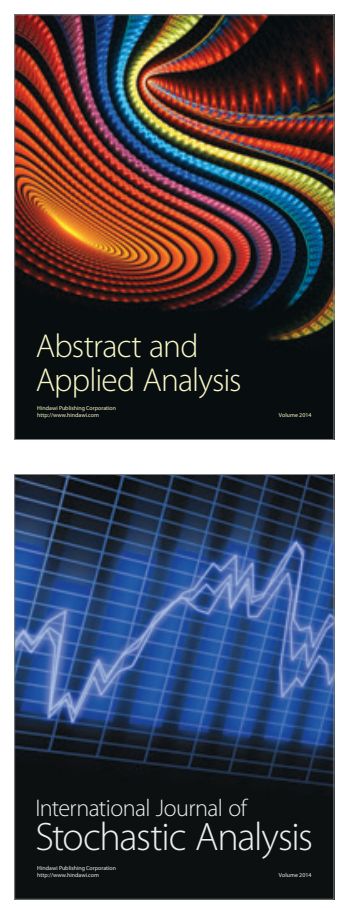

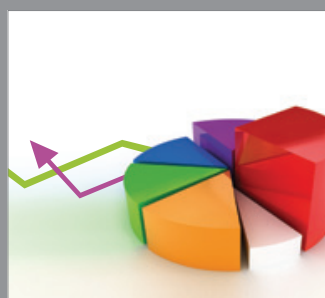

ournal of

Probability and Statistics

Promensencen
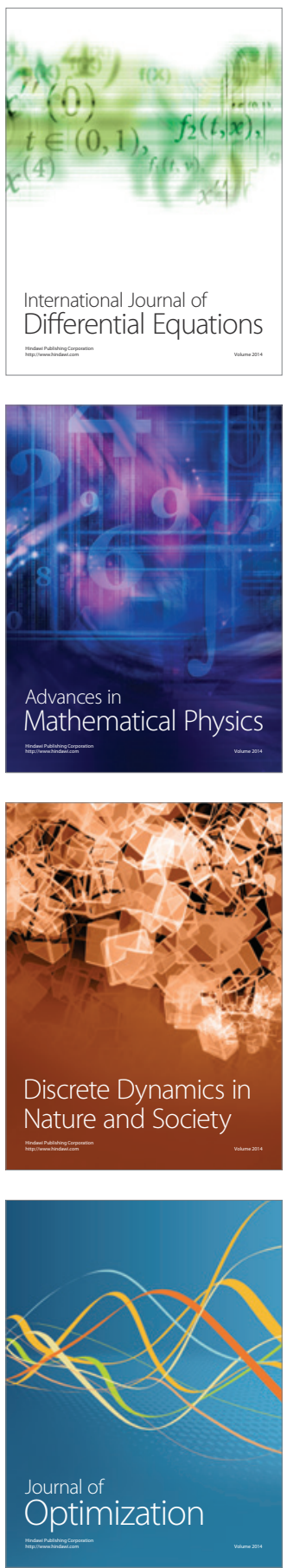